\title{
Síndrome de Gerstmann del Desarrollo Y TRASTORNOS EN LA ADQUISICIÓN DE LAS MATEMÁTICAS
}

\author{
DEVELOPMENTAL GERSTMANN SYNDROME \\ AND DISORDERS OF MATHEMATICS ACQUISITION
}

\author{
Sergio Dansilio (MD) \\ Universidad Católica del Uruguay
}

\begin{abstract}
Resumen: La tétrada caracterizada por una dificultad en el conocimiento práxico de los dedos, indistinción derecha/izquierda, discalculia y trastornos de la escritura (síndrome de Gerstmann) ha sido descrita en el desarrollo, probablemente vinculada a una perturbación madurativa del gyrus angular de manera bilateral o hemisférica derecha. En este trabajo se presentan tres casos con los tres elementos obligados de la tétrada y en un solo caso una perturbación vinculada a los niveles grafémico y ortográfico de la escritura. Dentro de la heterogeneidad de los casos, la perturbación en el dominio de las matemáticas se configura como una anaritmetia, caracterizada en base a un trastorno nuclear en la representación semántica del número. Se realizan las consideraciones teóricas acerca de la relación entre el conocimiento somestésico digital y la capacidad de representarse un espacio regularmente asimétrico, con la adquisición de las facultades matemáticas.
\end{abstract}

Palabras Clave: Sindrome de Gerstmann. Desarrollo. Neuropsicología. Discalculia.

\begin{abstract}
The tetrad characterized by a disturb in the praxic knowledge o the fingers, right-left disorientation, dyscalculia and dysgrafia (Gerstmann syndrome) has been described as a developmental disorder, probably linked to a dysfunction refered to the gyrus angularis whether bilateral or even in the right hemisphere and independent of dyslexia. In this paper three cases of a Developmental Gerstmann syndrome are presented, where the three obliged symptoms were founded, and only in one case graphemic and orthographic levels are affected. Among the heterogeneity of the cases, the disorder in the mathematical domain can be considered as an anaritmetia, that is, a perturbation in the nuclear representation of the number. Theoretical consideration are proposed about the relation in the acquisition between the somestesic discrimination of fingers, an asymmetric representational space, and mathematical abilities.
\end{abstract}

Key Words: Gerstmann Syndrome. Development. Neuropsychology. Dyscalculia.

\section{INTRODUCCIÓN}

Desde 1924 a 1930, Gerstmann describe un síndrome secundario a una lesión isquémica del gyrus angular izquierdo, caracterizado por una tétrada de síntomas: agnosia digital, indistinción derecha/izquierda, acalculia y agrafia (1940). La conjunción sintomática se consideró no casual, y fue interpretada como un trastorno circunscrito del esquema corporal, donde el conocimiento digital constituía el componente central del síndrome, y dio origen a la correlación topográfica entre el área del gryus angularis (circunvolución angular, área 39 de Brodmann) y dicho complejo sindrómico (Ardila, Concha \& Rosselli, 2000). Bajo esta concepción, se asoció el disturbio vinculado a los dedos al desarrollo de la capacidad de contar y a su uso para calcular (Ardila \& Rosselli, 2002). Una serie de hallazgos posteriores a las originales presentaciones de Gerstmann pusieron en cuestión el síndrome así concebido, ya sea que los síntomas se describían de manera aislada (falta de consistencia en la coexistencia sindrómica, la asociación casi constante de trastornos del lenguaje, indemnidad en algunos casos del gyrus angularis (Benton, 1987; 1992; Wingard et al, 2002). Sin embargo, estudios más recientes, tienden a confirmar la relación entre la 
capacidad de discriminar números y el conocimiento del cuerpo en adultos sanos que ya no utilizan sus dedos para resolver tareas matemáticas sencillas (Rusconi, Walsh, \& Butterworth, 2004). La estimulación magnética transcraneana sobre el gyrus angularis interrumpe pruebas que requieren un acceso al esquema digital, así como el procesamiento de magnitudes numéricas.

El síndrome de Gerstmann ha sido descrito en niños y adolescentes sin poderse remitir a una lesión cerebral adquirida, lo que indujo a acuñar el concepto del Síndrome de Gerstmann del Desarrollo (Kinsbourne \& Warrington, 1963; Kinsbourne, 1968; PeBenito et al, 1988; Benton, 1992; Suresh \& Sebastian, 2000). El Síndrome de Gerstmann del Desarrollo ha estado sin embargo vinculado a una disfunción hemisférica derecha o bilateral (Rourke, 1993; Suresh \& Sebastián, 2000). En estos casos, la disfunción, término vago, hace referencia a una perturbación en la fisiología de una área cerebral relativamente circunscrita, lo cual asienta sobre modificaciones microanatómicas (alteraciones sinápticas, de los patrones de conectividad, alteraciones de la normal estratificación de capas o en la migración neuronal). En ese sentido, desde estudios generales que exploran la relación entre dificultades del aprendizaje y niveles neurobiológico así como el más reciente modelo de casos con Síndrome de Turner han contribuido con evidencia parcial para realizar estas inferencias (Geschwind \& Galaburda, 1985 a y b; Rourke, 1993; Molko et al., 2004). Termina pues constituyendo una entidad diferente en cuanto a sus correlaciones encefálicas, aunque también en su significación clínica y probablemente en cuanto a los niveles cognitivos involucrados, ya que la relación entre los síntomas componentes se inscribe en un contexto distinto cuando las facultades matemáticas se están adquiriendo. Los trastornos de las facultades matemáticas informados en los trabajos citados son variados, en gran parte por lo variado también de su metodología y profundidad exploración, informándose desde una anaritmetia, perturbaciones en la determinación de la numerosidad, en la identificación de números, y en la secuenciación de números de acuerdo a su valor. La dificultad en las matemáticas en el síndrome de Gerstmann es independiente de un compromiso en la competencia psicolingüistica o en el dominio del código escrito (Rourke, 1993; Suresh \& Sebastián, 2000;
Kronenberger \& Dunn, 2003). Por otra parte, se ha visto que el síndrome de Gerstmann del desarrollo se solapa en gran medida con el Trastorno de Aprendizaje No Verbal, o Dificultad Visuoespacial del Aprendizaje (Rourke, 1993; Hardanek \& Rourke, 1994, Dansilio, 1999). Constituyen hallazgos frecuentes en el examen neurológico de estos chicos (signos neurológicos menores): Reflejos profundos asimétricos (vivos, policinéticos, aumento del área de obtención alternando con hiporreflexia), Babinski uni o bilateral, incoordinación motriz, agrafestesia, no discriminación de dos puntos, perturbaciones en el sentido de la posición del cuerpo, desorientación en la temporalidad, así como perturbaciones del comportamiento y en la capacidad de adaptación social. Los tres casos descritos en este trabajo, distintos inclusive en la edad, manifiestan más allá de la heterogeneidad, la consistencia en la coexistencia de los síntomas pero además una tendencia común en cuanto al género de trastornos centrales en la adquisición de las matemáticas.

\section{ESTUDIO DE CASOS}

Se presentan tres casos en los cuales se realiza el diagnóstico de Síndrome de Gerstmann del Desarrollo. Dichos casos, de manera separada, han sido publicados y descritos individualmente (Dansilio, 1999; Dansilio \& Chiapas, 1999). Más allá de que la metodología abordada en el estudio es diferente, ya que no solamente se trata de chicos de diferentes edades, sino que fueron captados en distintos momentos, se destacan algunos elementos en común. En todos los casos se siguió para la exploración de las facultades matemáticas la Batería Numerical (Gaillard, 2000), en aquel entonces aún no publicada, y en el desarrollo de los procesos de comprensión y transcodificación, se emplean números bien conocidos por controles sanos de igual escolaridad. Se sigue el modelo formal de McCloskey, Caramazza \& Basili (1985), los aporte subsiguientes de Macaruso, Harley y McCloskey (1992), y el formato del modelo de triple código de Dehaene y Cohen (1995). La exploración del conocimiento digital está basada en el estudio original de Kinsbourne \& Warrington (1962), y, siguiendo los principios de estos autores, se evita rigurosamente el componente verbal. En los casos Andrea y Roberto, se realizó el planteo de Trastorno por Déficit de Atención con Hiperactividad/Impulsividad, aunque no pueden 
extenderse las consideraciones ya que no se remitió el proceso y el mecanismo diagnóstico. La heterogeneidad de los casos cobra especial valor ante la consistencia de la asociación de los elementos fundamentales del Síndrome de Gerstmann, cuyo cuadro analítico puede observarse en la tabla 1.

\section{Caso Andrea}

10 años 6 meses. Sexo femenino. No-Diestra. Cursa $4^{\circ}$ año de Primaria. No posee antecedentes familiares neurológicos o psiquiátricos. No hay antecedentes patológicos pre o perinatales y el desarrollo de las conductas básicas se encuentra dentro de lo normal. De la exploración general se destaca un rendimiento intelectual descendido con valores del WISC 86 (Escala verbal), 74 (Escala realizativa), 78 (Global). Nivel operatorio (pruebas de conservación de cantidades físicas, según Piaget,), dentro de la eta- pa de operaciones concretas, con dominio de sustancia y solamente de manera parcial peso. No se encontraron trastornos específicos del lenguaje oral ni del dominio del código escrito. Hay, sin embargo, un disturbio de severa entidad en el domino visuoconstructivo (copia de figuras). El examen neurológico es informado normal. El EEG y la TAC de cráneo fueron normales.

La capacidad de discriminación derecha/izquierda, de acuerdo a la prueba Piaget-Head es correcta en su propio cuerpo, no en el oponente, ubicándose en un nivel de desempeño por debajo de los seis años. Igualmente está afectado el reconocimiento de dedos en pruebas no verbales. Andrea presenta una discalculia donde se compromete fundamentalmente la representación semántica de la cantidad, inclusive de números que logra transcodificar de manera adecuada, puede observarse la diferencia en la figura 1 donde se ven más comprometidas aquellas

Tabla 1.

Relación sinóptica de las características cínicas en los tres casos presentados

\begin{tabular}{|c|c|c|c|c|c|c|c|c|c|c|}
\hline $\begin{array}{l}0 \\
\mathscr{D}_{0} \\
\tilde{\delta}\end{array}$ & $\begin{array}{l}\overline{0} \\
\frac{\pi}{0} \\
\text { Un }\end{array}$ & $\begin{array}{l}0 \\
\dot{x} \\
0 \\
c\end{array}$ & Discalculia & $\begin{array}{c}\text { Dificultad en } \\
\text { Reconocimiento } \\
\text { Digital }\end{array}$ & $\begin{array}{l}\text { Indistinción } \\
\text { Derecha } \\
\text { Izquierda }\end{array}$ & $\begin{array}{l}\text { Disturbios en el } \\
\text { código escrito }\end{array}$ & $\begin{array}{c}\text { Trastornos } \\
\text { visuoconstructivos }\end{array}$ & Dislexia & $\begin{array}{c}\text { Otros } \\
\text { diagnósticos }\end{array}$ & \\
\hline 1 & 10.6 & $F$ & + & + & + & - & + & - & TDAH & - \\
\hline 2 & 15 & M & + & + & + & - & + & - & TDAH & - \\
\hline 3 & 25 & $F$ & + & + & + & + & - & - & - & - \\
\hline
\end{tabular}

Figura 1

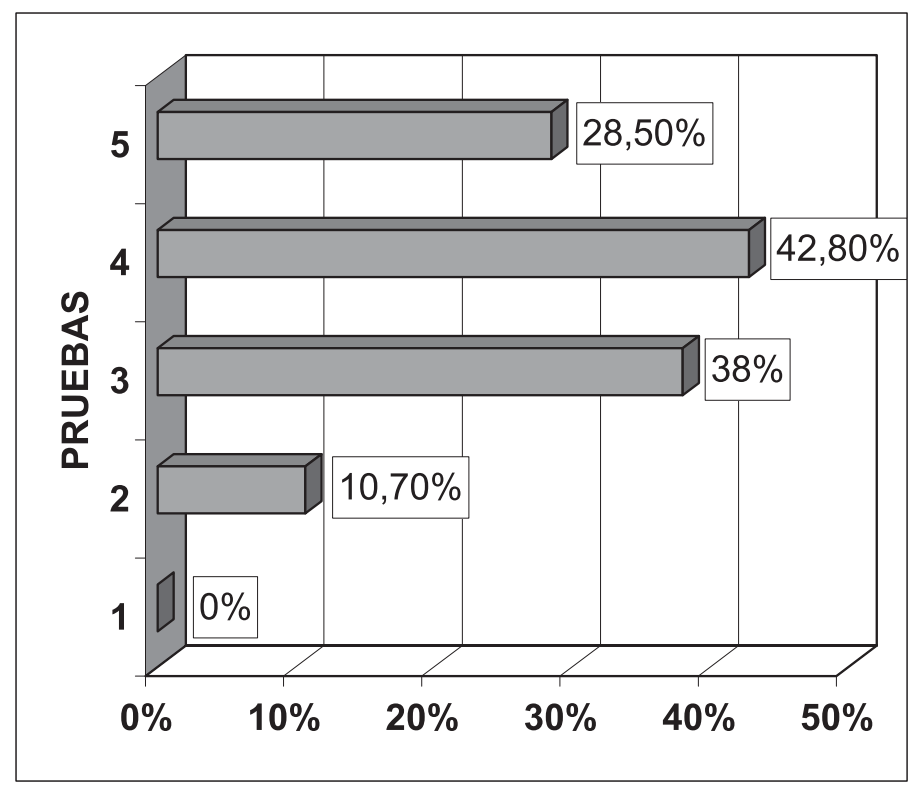

REFERENCIAS. (1) Lectura en código alfabético. (2) Lectura en código arábigo. (3) Lectura desde el ábaco. (4) Comparación de magnitudes (ambos códigos). (5) Sucesión en código arábigo ("antes - después") 
pruebas que requieren un mayor grado de procesamiento semántico. Así en la lectura desde el ábaco, o en las pruebas de comprensión comete un mayor porcentaje de errores, pruebas todas con efecto piso. Puede transcodificar de manera adecuada números como 2015 , /dos mil quince/, números que luego no logra discriminar en su magnitud (mayor o menor). La estimación perceptiva de la magnitud ("20 niños en una escuela es... mucho, poco, adecuado"; estimación de tiempo restante y duración; etc.), y la capacidad de operar con cantidades están también comprometidas, así como la capacidad de operar ya en sumas de mínima dificultad. No lograba aprender la lectura del reloj. Se trata, entonces de una anaritmetia.

\section{Caso Roberto}

15 años. Sexo masculino. Zurdo (zurdería familiar). $6^{\circ}$ Primaria (repite 1er. Año). Procede de parto inducido - a término (Hipertensión del embarazo) PN 3.650. Adquisición de conductas básicas normal (salvo caminar: 18 meses). Recibió comitoína a partir de los 2 años y durante 4 años por EEG "patológico", sin crisis epilépticas. A los 9 años el neuropediatra indica metilfenidato por diagnóstico de TDAH probable (datos de la historia clínica, no se informa procedimiento de diagnóstico). El EEG y la TAC de cráneo actua- les son normales. No recibe medicación. Derivación al Departamento de Neuropsicología con diagnóstico de "discalculia". La exploración general (nivel intelectual, WAIS y Raven, nivel operatorio) muestra valores dentro de la media para edad y escolaridad. En el estudio neuropsicológico no se constatan perturbaciones en los dominios ejecutivo, mnésico, lenguaje oral o código escrito. Hay un disturbio moderado en el dominio visuoconstructivo, con una figura compleja de Rey-Osterrieth en el percentil 50 (realización tipo IV), pero mostrándose incapaz de reproducir la perspectiva, y simplificando figuras.

De la exploración de las facultades matemáticas se destaca un patrón que reproduce la hallado en el caso previo. Está afectadas las pruebas que evalúan la comprensión (en este caso no se realizó la lectura desde el ábaco), y en la medida que la prueba involucra un mayor componente semántico. En este caso logra transcodificar numerales en el rango de la decena de mil, con ceros sintácticos, numerales que después no discrimina de manera adecuado en pruebas de comparación mayor/menor, o en completar y determinar la corrección de pruebas de sucesión. Tanto las pruebas de estimación perceptiva de la cantidad como la capacidad de operar se encuentran alteradas con respuestas aleatorias.

Figura 2.A.

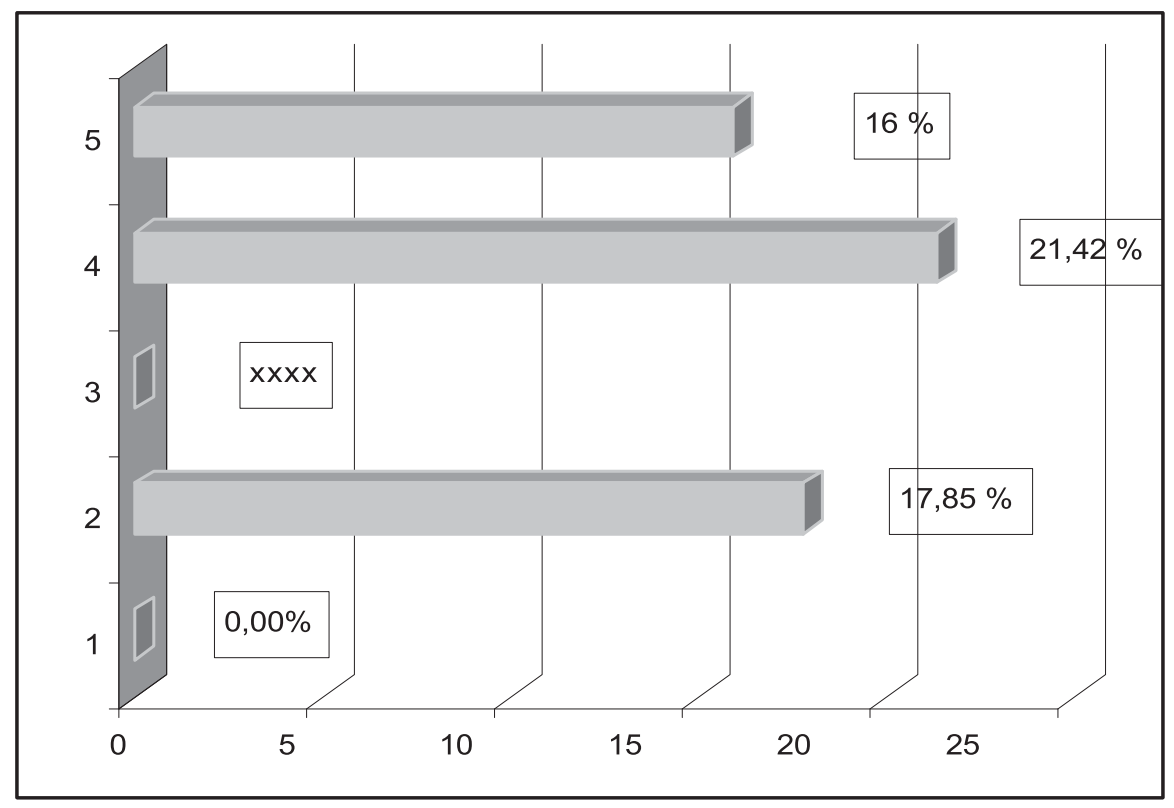

REFERENCIAS. (A) Pruebas de comprensión / producción. (1) Lectura en código alfabético. (2) Lectura en código arábigo. (3) Lectura desde el ábaco. (4) Comparación de magnitudes (ambos códigos). (5) Sucesión en código arábigo ("antes - después"). (B) Alineamiento de números según su valor. 
Figura 2.B.

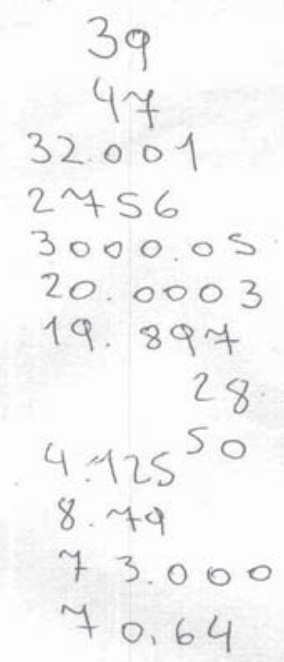

Figura 3

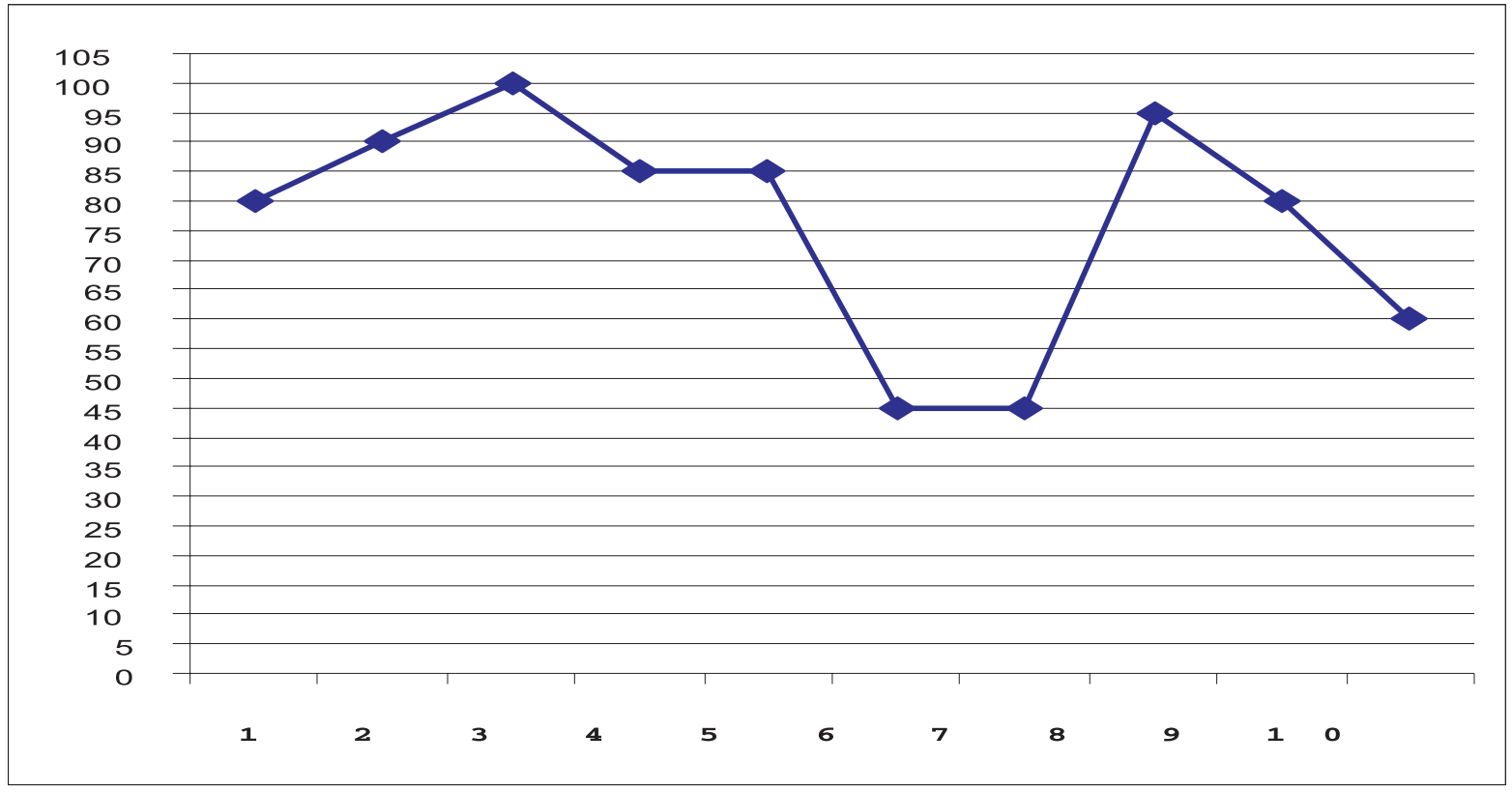

Caso Claudia. Batería Numerical, la cual con baremos para 8-9 años de edad. (1) Escala cuantitativa (2) Factor digital (3) Factor oral (4) Factor espacial (5) Factor analógico (6) Factor cálculo oral-escrito (7) Factor cálculo escrito (8) Factor alfabético (9) Factor estimación/proposición (10) Escala cualitativa.

\section{Caso Claudia}

25 años, sexo femenino. Diestra homogénea $2^{\circ}$ Secundaria. Nace pretérmino (24 semanas). $\mathrm{PN}<1000 \mathrm{gr}$ (bajo peso) (internación en $\mathrm{CTI}$ ). Convulsiones en apirexia, EEG que mostró foco irritativo central izquierdo, recibiendo tratamiento con fenobarbital. Remisión de crisis al año. Desarrollo de conductas básicas (estática y marcha autónomas, esfínteres, prime- ras palabras y frases) dentro de edades apropiadas. TAC de cráneo actual normal. Cursa Primaria sin repeticiones. Se le caracteriza como "distraída". Sin indicadores de hiperquinesia o impulsividad. Dificultades generales y especialmente en matemáticas, requiere apoyo pedagógico especial. Buen desempeño en idioma español y lengua extranjera (inglés). No se destacan faltas de ortografía. 
Al ingresar en Secundaria se hace más invalidante la dificultad en matemáticas. Repite $1^{\circ}$ año. La joven relata además dificultades referidas dentro de dominios no-matemáticos como articular pasos de un razonamiento, generar consistencia en una serie de contenidos, establecer (reproducir) el orden de una narración, aprendizaje de series.

Evocación adecuadas (Protocolo Montevideo). En el examen general (WAIS, Raven), así como en la exploración neuropsicológica (dominio ejecutivo, mnésico, lingüístico) no se encuentran alteraciones específicas o globales con rendimiento dentro de la media. Se excluye, por supuesto, el subtest de matemáticas del WAIS. No se evidencian perturbaciones de naturaleza visuoconstructiva en la copia de figuras, a diferencia de los casos anteriores. Presenta, en cambio, un trastorno en el nivel grafémico y ortográfico de la escritura, con errores de secuenciación grafémica y de tipo disortográfico.

Hay un descenso severo en la discriminación derecha/izquierda, en la prueba de PiagetHead. Se acompaña de una perturbación en la capacidad de discriminar dedos, especialmente en aquellas tareas de mayor carga somestésica y sin control visual. La discalculia hallada. En la exploración del cálculo surge un patrón diferente, encontrándose los procesos de transcodificación conservados. Las perturbaciones radican en la capacidad de realizar cálculos mentales y cálculos escritos, pero tam- bién en la escala cualitativa, donde se computan estimaciones que exploran el conocimiento referencial de la magnitud. Está alterada la realización de pruebas de sucesión que se realizaron de acuerdo a la hipótesis de que la paciente poseía una dificultad en la representación de series (que se extiende para series no numéricas), con un porcentaje de errores de $75 \%$ (controles sanos, efecto piso, normal sin errores). Se encuentran resultados tales como: $\underline{39000} 40000 \quad 41000$. Las pruebas de sucesión exploran la comprensión del número de su dimensión ordinal, aunque están muy marcadas por el componente sintáctico. En la figura 4 se muestra la realización de un cálculo escrito por Claudia. A lo largo del desarrollo, los trastornos en la representación semántica no alcanzan a incidir mayormente en los procesos de transcodificación, aunque si condicionan la capacidad de operar con números y en poder ubicarlos en una métrica serial. Reúne también las características de una anaritmetia.

\section{DISCUSIÓN}

En los tres casos, está afectada de manera nuclear la representación semántica del número, lo cual se refleja en una dificultad para realizar pruebas de comprensión bajo diferentes modalidades, en el supuesto de que el sistema semántico es único. Esto contrasta con pro-

Figura 4

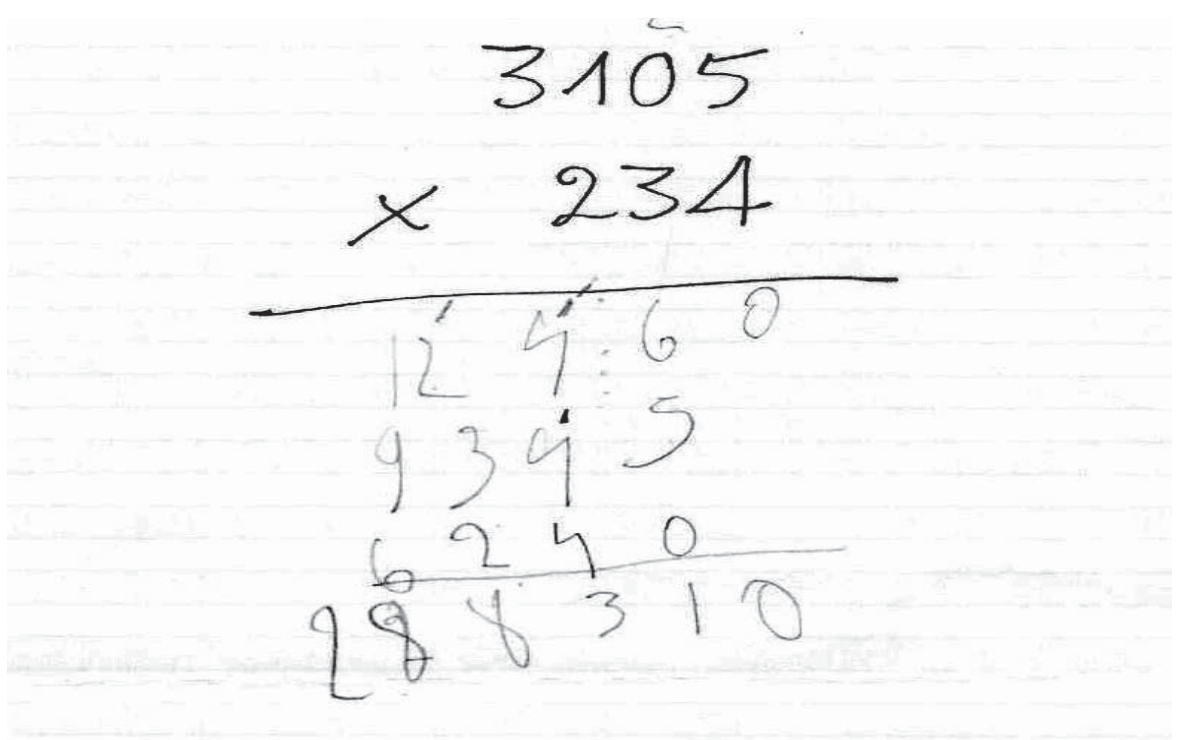

Caso Claudia. Realización de una multiplicación escrita. Los errores de alineamiento fueron explicados en función del conocimiento de las relaciones entre las cifras que hacían a los productos intermedios. 
cesos de transcodificación relativamente indemnes (transcodifica de manera correcta numerales cuya magnitud no se logra discriminar). En los casos de Andrea y Roberto, se compromete la capacidad de realizar estimaciones perceptivas de la cantidad, lo cual condiciona una serie de preguntas acerca de la relación entre la capacidad de entender el espacio bajo una métrica y las facultades matemáticas. Es de destacar que las pruebas de transcodificación que utilizan el ábaco están más afectadas (mayor número de errores) ya que, al constituir pruebas novedosas, requieren una elaboración semántica necesaria para reconstruir el otro código a partir de una presentación prácticamente prictográfica. La dificultad para adquirir la estructura de las operaciones básicas es subsidiaria de este déficit nuclear, constituyendo una verdadera anaritmetia.

El caso Claudia ofrece una serie de diferencias, más allá de la edad. Se encuentra un déficit en la capacidad de discriminar y reproducir secuencias ordenadas (series). No puede adscribirse a un déficit inespecífico en la capacidad de "secuenciar" (en el caso de que tal cosa exista), ya que logra extraer de manera adecuada sucesiones de figuras o patrones no verbalizables y de anticiparlos. Las series no solamente implican la noción de precesión y sucesión, sino una relación de naturaleza conjuntística. Es en este nivel donde se produce el disturbio. En qué medida o cuál es la razón causal que pudiera emparentar el trastorno en la seriación con la dificultad en la representación numérica no fue posible de determinar en ese primer estudio. De manera fenomenológica, puede sin embargo sostenerse, que el trastorno en la capacidad de establecer una serie afecta uno de los componentes que hacen a la noción de número de acuerdo a Piaget \& Szeminska (1975), pudiendo entonces encontrarse en la génesis de la discalculia. Otra vez, la dificultad en la representación semántica del número (en este caso más que de la cardinalidad, de la ordinalidad), perturba la adquisición de las operaciones muy parcialmente compensadas a lo largo de la educación. Se trata pues, de una anaritmetia en el sentido de un déficit en el concepto nuclear del número (Butterworth, 2005), que condiciona una perturbación en la capacidad de manejar la realidad en términos cuantitativos, como se puede inferir de Ardila \& Rosselli (2002).
Ardila \& Rosselli, entendiendo también la anaritmetia como la acalculia primaria de Berger, la describen como un déficit básico en la habilidad de computar o realizar operaciones matemáticas no secundario a disturbios lingüísticos, espaciales o de tipo frontal (2002). De acuerdo a Ardila y Rosselli los pacientes con anaritmetia presentan: Una pérdida de los conceptos numéricos, imposibilidad de entender cantidades, defectos para utilizar algoritmos en los cálculos (lo que los autores Ilaman "reglas sintácticas" de las operaciones) y fallas en la comparación de números. Estos pacientes pueden ser capaces de contar en voz alta o extraer datos básicos (tablas). El déficit se constata tanto en el cálculo oral como escrito ya que constituye un trastorno fundamental que afecta toda modalidad, pudiendo ser interpretada como un defecto en entender la forma en la cual el sistema numérico opera. La anaritmetia se observa en adultos, en casos de lesiones del gyrus angularis izquierdo, es decir, que ha podido ser correlacionada con una topografía discreta del cerebro.

Butterworth ha sostenido la existencia de un déficit específico en una capacidad muy básica para el entendimiento de los números, la cual conduce a la gama de perturbaciones que se encuentran luego en el aprendizaje de las matemáticas (2005). La propuesta de Butterworth está basada en la existencia de una competencia genéticamente determinada para el reconocimiento y la manipulación mental de numerosidades (definida como la idea básica de los valores cardinales de un conjunto). Los trastornos selectivos se generarían cuando esta competencia específica, que Butterworth llama módulo del número, no se desarrolla normalmente: es lo que denomina la "hipótesis del módulo numérico deficitario". A diferencia de lo postulado por Gallistel y Gelman, por ejemplo (2000), el conocimiento numérico y el conocimiento del lenguaje siguen en su génesis desarrollos independientes. Butterworth defiende además la probabilidad de que exista un sistema anatómico cerebral discriminado que está afectado en las discalculias (2005), ya sea empleando evidencias relativamente directas como las de Isaacs et al. (2001), o mediante la amplia serie de documentos imagenológicos y fisiológicos que se han publicado acerca de las relaciones entre las competencias aritméticas y zonas circunscritas del cerebro (Stanescu-Cosson et al, 2000; Dehaene et al, 2003; Kong et al, 
2005; Rivera et al, 2005). La anaritmetia, como producto de un déficit primario, constituye el resultado del trastorno nuclear en el módulo del número de Butterworth.

La indistinción derecha/izquierda y el trastorno en el reconocimiento de los dedos, perturban el proceso de desarrollo en el dominio de las facultades matemáticas. La matematización del espacio, requiere de una espacio anisotrópico y ordenado, muy inestable al fallar la lateralización habitual del mismo. El conocimiento práxico de los dedos constituye la herramienta material sobre la cual se maneja literalmente la cantidad, el número. La capacidad de discriminación digital parece ser un elemento pronóstico de la adquisición de las matemáticas, independiente del nivel intelectual general (Fayol, Barrouillet \& Marinthe, 1998). Que en adultos con las facultades adquiridas tanto la agnosia digital como la confusión derecha-izquierda no provoquen necesariamente una acalculia constituye un dato que no puede extenderse para el desarrollo. El adecuado desarrollo de la praxia digital, y el manejo de un espacio asimétrico y estable, son condiciones para la adquisición del domino, y de ahí que su compromiso afectará el aprendizaje de las matemáticas en un sentido profundo y desde el inicio de la representación semántica del número. La comprensión del Síndrome de Gerstmann del Desarrollo abre una ventana para entender la interacción entre las diversas dimensiones representacionales que hacen a la génesis del número, pero también para generar medidas de prevención y de reeducación cuando el problema se presenta. Ante cada caso de un trastorno del cálculo, entonces, es imprescindible obtener un adecuado análisis de la capacidad de discriminar el espacio asimétrico, y de la capacidad de conocimiento somestésico y práxico de los dedos. Permanece aún por explicar el problema de la disgrafia, aunque se trata del síntoma inconstante en la tétrada. Los trastornos de la escritura son variados y heterogéneos en cuanto a los niveles de procesamiento que se afectan (Kinsbourne \& Warrington, 1963 y 1968; PeBenito et al, 1988; Benton, 1992; Suresh \& Sebastian, 2000). Incluyen errores disortográficos, errores de nivel grafémico (secuenciales, que son característicos, pero también omisiones o sustituciones), alográficos y del grafismo (disgrafía propiamente dicha con transposición de letras o letras malformadas). Esta heterogeneidad ha hecho imposible que se pueda establecer un vínculo funcional entre los trastornos en la adquisición de la escritura y el resto de los componentes del síndrome. La asociación de trastornos en las habilidades visuoconstructivas y las dislexias aunque pueden encontrarse, y particularmente los primeros, son muy inconstantes (PeBenito et al, 1988; Surresh \& Sebastian, 2000). En el caso CR se observan perturbaciones de diferente tipo, aunque de nivel central (niveles del grafismo y alográfico preservados). Finalmente, la relación con una disfunción de áreas circunscritas del cerebro (en este caso, el gyrus cingularis izquierdo, derecho o bilateral), y siguiendo las precisiones realizadas en la introducción, es fundamentalmente inferencial e indirecta, fundamentada en la coexistencia comparativa de los síntomas y hallazgos en modelos típicos, probablemente asociadas a trastornos microanatómicos que afectan la normal fisiología de las redes involucradas en estas funciones.

\section{CONCLUSIONES}

El síndrome de Gerstmann, vinculado al gyrus angularis izquierdo y originalmente descrito en casos de pacientes adultos con lesión cerebral, ha sido encontrado como trastorno del desarrollo, probablemente vinculado a una disfunción madurativa de áreas similares aunque de manera bilateral o incluso lateralizada a derecha. La disfunción madurativa responde a un disturbio en el funcionamiento del área involucrada, y vinculada a micodisgenesias aún no bien determinadas. No constituye un hallazgo raro cuando se lo busca. Aunque es necesaria mayor investigación clínica y experimental, así como una correcta sistematización de la evaluación y un adecuado nivel de detalle para la exploración, existen argumentos a favor de una relación psicogenética entre la capacidad de discriminar dedos, la estabilidad de la distinción derecha/izquierda, y la adquisición de las facultades matemáticas básicas. Esta relación puede articularse con la maduración de sistemas neurofuncionales discriminados, y es en este contexto donde se entiende el valor teórico de la tétrada clínica que caracteriza al síndrome de Gerstmann. Es necesario avanzar también, en establecer relaciones más adecuadas entre el nivel del desarrollo específico y diferenciado del encéfalo, y los hallazgos neuropsicológicos descritos. 


\section{REFERENCIAS}

Ardila, A; \& Rosselli, M. (2002). Acalculia and Dyscalculia. Neuropsychology Review, 2, 179-230.

Ardila, A; Concha, M; \& Rosselli, M (2000). Angular gyrus sindrome revisited: Acalculia, finger agnosia, right-left disorientation, and semantic aphasia. Aphasiology, 14, 743-754.

Benton, A. (1987). Mathematical disability and the Gerstmann syndrome. En: G. Deloche \& X. Seron (Eds) Mathematical Disabilities: A Cognitive Neuropsychological Perspective (pp. 111-120). Erlbaum: Hillsdale.

Benton, A. (1992). Gerstmann's syndrome. Archives of Neurology, 49: 445-447.

Butterworth, B. (2005). Developmental dyscalculia. En: Jamie ID Campbell (Ed), The Handbook of Mathematical Cognition (pp. 455-467). New York: Psychology Press.

Dansilio, S. (1999). Dificultad no verbal del aprendizaje y discalculia. Presentado en: $6^{\circ}$ Congreso de la Sociedad Latinoamericana de Neuropsicología. Varadero, Cuba.

Dansilio, S. \& Chiappas, M. (1999). Representación semántica del número y procesos de transcodificación en un caso de discalculia. $R e$ vista Latina de Pensamiento y Lenguaje y Neuropsychologia Latina, 1: 49-64.

Dehaene, S.; \& Cohen, L. (1995). Towards an anatomical and functional model of number processing. Mathematical Cognition, 1, 83-120.

Fayol, M; P Barrouillet,P; \& C Marinthe, C. (1998). Predicting arithmetical achievement from neuropsychological performance: A longitudinal study. Cognition, 1998, 68, 63-70.

Gaillard, F. (2000). Numerical. Test neurocognitif pour l'apprendtissage du nombre et du calcul. Université de Lausanne, Laussane: Institut de Psychologie.

Gallistel, C. R.; \& Gelman, R. (2000). Non-verbal numerical cognition: From reals to integers. Trends in Cognitive Sciences, 4(2), 59-65.

Gerstmann, J. (1940). Syndrome of finger agnosia, disorientation for right and left, agraphia and acalculia. Archives of Neurology and Psychiatry, 44, 398-408.

Geschwind N; \& Galaburda AM (1985a). Cerebral Lateralization: Biological meschanisms, associations, and pathology: I. A hypothesis and a program of research. Archives of Neurology, 42: 428-455.

Geschwind N ; \& Galaburda AM (1985b). Cerebral Lateralization: Biological mechanisms, associations, and pathology: II. A hypothesis and a program of research. Archives of Neurology, 42: 428-455.

Hardanek, M.C.S.; \& Rourke, B.P. (1994). Principal identifying features of the syndrome of nonverbal learning disabilities in children. Journal of Learning Disabilities, 3: 144-154.
Isaacs E.B., Edmonds C.J., Lucas A.; \& Gadian, D.G. (2001). Calculation difficulties in children of very low birthweight: A neural correlate. Brain 124, 1701-1707.

Kinsbourne, M. (1968). The developmental Gerstmann syndrome. Pediatric Clinics of North America, 15, 771-778.

Kinsbourne, M.; Warrington, E.K.(1962). A study of finger agnosia. Brain, 85, 47-66

Kinsbourne, M \& Warrington, EK. (1963). Developmental Gerstmann's syndrome. Archives of Neurology, 8, 490-501.

Kong, J.; Wang, Ch.; Kwong, K.; Vanguel, M.; Chua, E.; \& Gollub, R. (2005). The neural substrate of arithmetic operations and procedure complexity. Cognitive Brain Research, 22, 397-405.

Kronenberger, W.G.; \& Dunn, D.W. (2003). Learning disorders. Neurolologic Clinics, 21(4), 941-52.

Macarusso, P; Harley, W \& McCloskey, M (1992). Assesment of acquired dyscalculia. En: D. I. Margolin (Ed) Cognitive Neuropsychology in clinical practice pp. 405-434). Oxford University Press: New York.

McCloskey, M.; Caramazza, A. \& Basili, A. (1985). Cognitive mechanisms in number processing and calculation: Evidence from dyscalculia. Brain and Cognition 4, 171-196.

Molko, N; Cachia, A; Riviere, D; Mangin J. F.; Bruandet, M.; LeBihan, D; Cohen, L; \& Dehaene, S. (2004). Brain anatomy in Turner Syndrome: Evidence for impaired and spatial numerical networks. Cerebral Cortex 14: 840-850.

PeBenito, R.; Fisch, C.; \& Fisch, M.L. (1988). Developmental Gerstmann's syndrome. Archives of Neurolology, 45, 977 - 982.

Piaget, J.; \& Szeminska, A. (1975). Génesis del número en el niño. Buenos Aires: Biblioteca Pedagógica Guadalupe.

Piaget, J. (1991). Psicología de la inteligencia. Buenos Aires: Ediciones Siglo Veinte. (Original de 1950).

Rivera, S.M.; Reiss, A.L.; Eckert, M.A.; \& Menon, V. (2005). Developmental changes in mental arithmetic: Evidence for increased functional specialization in the left inferior parietal cortex. Cerebral Cortex, 15, 1779-1790.

Rourke BP (1993) Arithmetic disabilities, specific and otherwise: A neuropsychological perspective. Journal of Learning Disabilities 4: 214-226.

Rusconi, E; Walsh, V; \& Butterworth, B (2005). Dexterity with numbers: rTMS over left angular gyrus disrupts finger gnosis and number processing. Neuropsychologia, 43: 1609-1624.

Stanescu-Cosson, R.; Pinel, P.; van de Moortele, PF.; Le Bohan, D.; Cohen, L.; \& Dehaene, S. (2000). Understanding dissociations in dyscalculia. A brain imaging study of the impact of number size on the cerebral networks for exact and approximate calculation. Brain, 123, 22402255. 
Suresh, P.A. \& Sebastian, S. (2000). Developmental Gerstmann's syndrome: A distinct clinical entity of learning disabilities. Pediatric Neurology, 4: 267-278.
Wingard, E.M., Barrett, A.M., Crucian, G.P., Doty, L., \& Heilman, K.M. (2002). The Gerstmann syndrome in Alzheimer's disease. Journal of Neurology, Neurosurgery and Psychiatry, 72(3): 403-405. 